\title{
Thyroid hormone for treatment-resistant depression: A systematic review and meta-analysis
}

R. Lorentzen, BMSc1, J. N. Kjar, MD2

S. D. Østergaard, MD, PhD2, M. M. Madsen, MD2 1 Aarhus University, Denmark

2Aarhus University Hospital - Psychiatry, Aarhus, Denmark

\section{BACKGROUND}

- Treatment-resistance is of significant clinical concern, affecting approximately $40 \%$ of patients with unipolar depression

- Augmentation with thyroid hormones is used in the management of treatment-resistant depression (AUH has clinical guidelines), however, the evidence for the effect of thyroid hormones is not fully established

- We strive to systematically review the effect of thyroid hormone treatment in the management of treatmentresistant unipolar depression

\section{METHODS}

- We follow the PRISMA guideline for systematic reviews and meta-analysis

- Searches were carried out in the MEDLINE (PubMed), EMBASE and PsycINFO databases

- Each record was screened independently by at least two authors. Consensus was reached through discussion

- Data extraction will be performed independently by at least two authors followed by statistical analysis

\section{INCLUSION}

- Prospective, controlled studies of treatment with thyroid hormone of unipolar treatment-resistant depression

- No limitations on type of control intervention/treatment

\section{RESULTS}

- The search yielded $\mathbf{1 3 2 3}$ records, of which 418 were duplicates

- 9 studies remained after screening and full-text assessment

- A total of 639 patients in 9 studies

- Data extraction is currently underway and will be followed by assessment of bias and confounders for each study and meta-analysis

\section{CONCLUSION}

We expect that the results from the metaanalysis of the trials identified in this systematic review will be very useful to psychiatrists managing treatment-resistant depression.

\section{Co-medication type}

-TCA $\backsim$ SSRI $\square$ Various

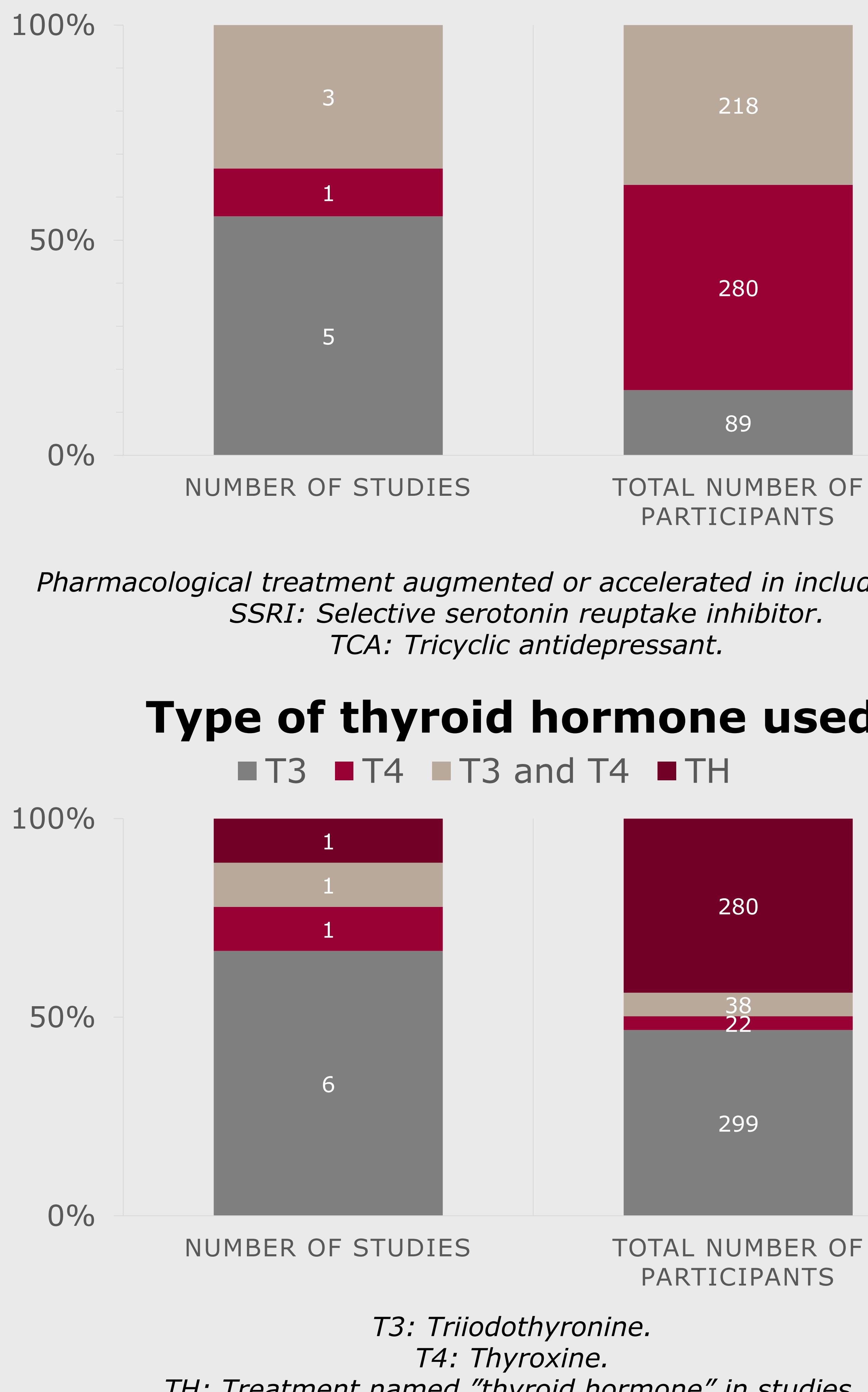

Flow-chart of study selection process

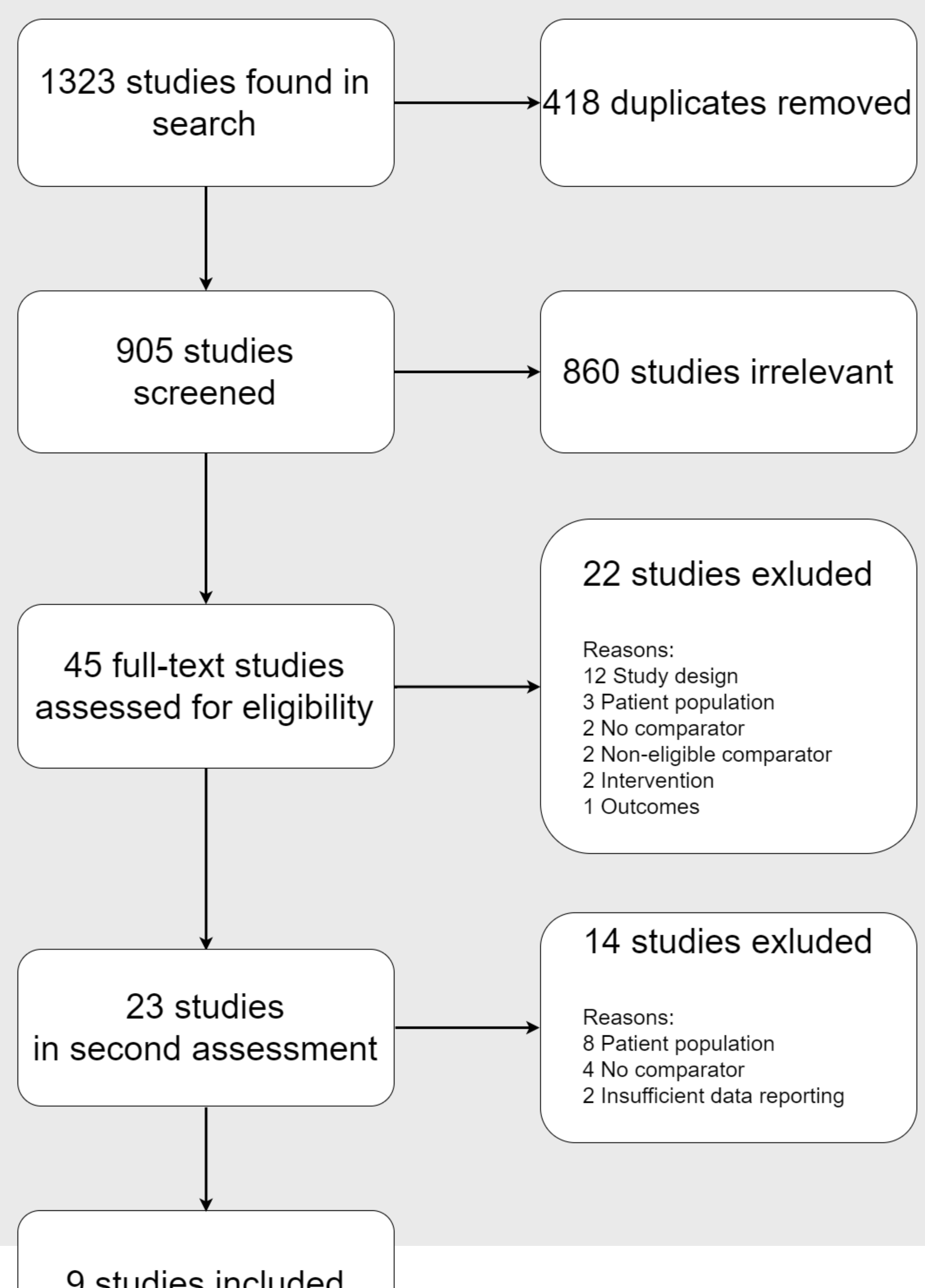

